\title{
Secondary Immune Thrombocytopenia in Metastatic Renal Cell Carcinoma: A Case Report and Discussion of the Literature
}

\author{
Dillon C. Cockrella Raj S. Kasthuri ${ }^{a, b, c}$ Ersan Altun $^{d}$ Tracy L. Rose ${ }^{a, c}$ \\ Matthew I. Milowsky ${ }^{a, c}$ \\ aDepartment of Medicine, University of North Carolina School of Medicine, Chapel Hill, \\ NC, USA; bBlood Research Center, University of North Carolina, Chapel Hill, NC, USA; \\ 'Lineberger Comprehensive Cancer Center, University of North Carolina, Chapel Hill, NC, \\ USA; dDivision of Abdominal Imaging, Department of Radiology, University of North \\ Carolina School of Medicine, Chapel Hill, NC, USA
}

\section{Keywords}

Immune thrombocytopenia - Renal cell carcinoma - Paraneoplastic syndrome ·

Thrombopoietin receptor agonists

\section{Abstract}

Immune thrombocytopenia (ITP) is a rare paraneoplastic syndrome of solid tumor malignancies. In previously described cases of renal cell carcinoma (RCC) associated with secondary ITP, treatment has consisted of nephrectomy, splenectomy, and corticosteroids. Here, we describe a case of metastatic RCC presenting with a right ventricular mass and subsequent development of secondary ITP. The clinical course was complicated by recurrent severe thrombocytopenia despite treatment with corticosteroids, rituximab, and thrombopoietin receptor agonists, precluding cancer-directed therapy and anticoagulation. Further study is needed to determine the optimal management strategy for malignancy-associated ITP.

\section{Introduction}

Immune thrombocytopenia (also referred to as immune thrombocytopenic purpura or ITP) is an autoimmune bleeding disorder characterized by isolated low platelet counts resulting from pathologic immune-mediated mechanisms. It occurs as a result of antibodymediated targeting of platelet antigens in the setting of normal erythrocyte and leukocyte function without evidence of impaired bone marrow production. ITP is a common cause of

\section{Karger ${ }^{\prime \prime}=$}


thrombocytopenia in both children and adults, with an estimated incidence of 1.9-6.4 per 100,000 children/year and 1.6-3.9 per 100,000 adults/year [1]. Primary ITP is a diagnosis of exclusion, where no disorder or medication associated with thrombocytopenia is identified, whereas secondary ITP refers to thrombocytopenia attributable to an underlying disease or the development of drug-induced thrombocytopenia [2].

Although secondary ITP is more commonly associated with rheumatologic disorders, infections, and hematologic malignancies, it is also seen in patients with solid tumor malignancies, with the highest reported cases in lung and breast cancers [3]. Approximately 20\% of patients with renal cell carcinoma (RCC) experience some form of a paraneoplastic syndrome; however, secondary ITP associated with RCC is rare [4]. Removal of the primary tumor, corticosteroids, and splenectomy have been used to treat RCC-associated ITP with variable success [3]. Further, clinical data supporting effective therapies for patients with malignancy-associated ITP who are not surgical candidates is lacking. In this report, we describe a challenging case of secondary ITP in a patient with advanced RCC.

\section{Case Presentation}

A 47-year-old female with a past medical history of chronic obstructive pulmonary disease, depression, and tobacco use presented with chest pain and hemoptysis. Computed tomography (CT) angiogram revealed a large thrombus extending from the right ventricular (RV) cavity into the main pulmonary artery with additional bilateral segmental and subsegmental pulmonary emboli and evidence for right heart strain. Anticoagulation with unfractionated heparin was initiated, and the patient was transferred to a tertiary medical center for further management.

Transthoracic echocardiogram (TTE) revealed a large RV mobile thrombus $(2.4 \times 1.5 \mathrm{~cm})$ extending from the right ventricle through the outflow tract into the main pulmonary artery with associated moderate RV systolic dysfunction. She subsequently underwent intracardiac tumor resection, pulmonary artery embolectomy, and tricuspid valve ring repair. Surgical pathology demonstrated unorganized to early organizing thrombus with prominent clusters of highly atypical cells with enlarged nuclei and atypical mitotic figures. Immunohistochemistry was positive for pancytokeratin, PAX-8, and CD10. Overall, the findings were consistent with a cytologically high-grade, pleomorphic tumor favoring metastatic RCC. Follow-up TTE revealed continued moderate RV systolic dysfunction though significant decrease in the size and mobility of the RV mass. Her postoperative course was complicated by anemia and volume overload. She was transitioned from unfractionated heparin infusion to warfarin and aspirin prior to discharge.

Abdominal CT scan obtained as an outpatient revealed a 9.9-cm left renal mass, multiple associated enlarged retroperitoneal lymph nodes, moderate pericardial effusion, nodular and peripheral wedge-shaped opacities in the lung bases concerning for metastatic disease or pulmonary infarcts, and small right greater than left pleural effusions with pleural thickening concerning for malignant effusions.

She was re-admitted after a syncopal event, and chest CT revealed multiple bilateral pulmonary emboli and a $5.5 \times 3.0 \mathrm{~cm}$ RV mass with severely reduced RV function. Brain magnetic resonance imaging (MRI) scan revealed multiple cortical $2-3 \mathrm{~mm}$ foci of enhancement involving the bilateral frontal lobes concerning for metastases. Abdominal MRI was notable for retroperitoneal lymphadenopathy and increasing size $(10.1 \times 7.6 \times 10.8 \mathrm{~cm}$; Fig. 1$)$ of the left renal mass without evidence of invasion of surrounding organs. Anticoagulation was continued on admission given concern that the pulmonary emboli in part were related to bland thrombus formation in addition to tumor emboli. Further cardiac surgery, vascular

\section{Karger's}




\section{Case Reports in Oncology}

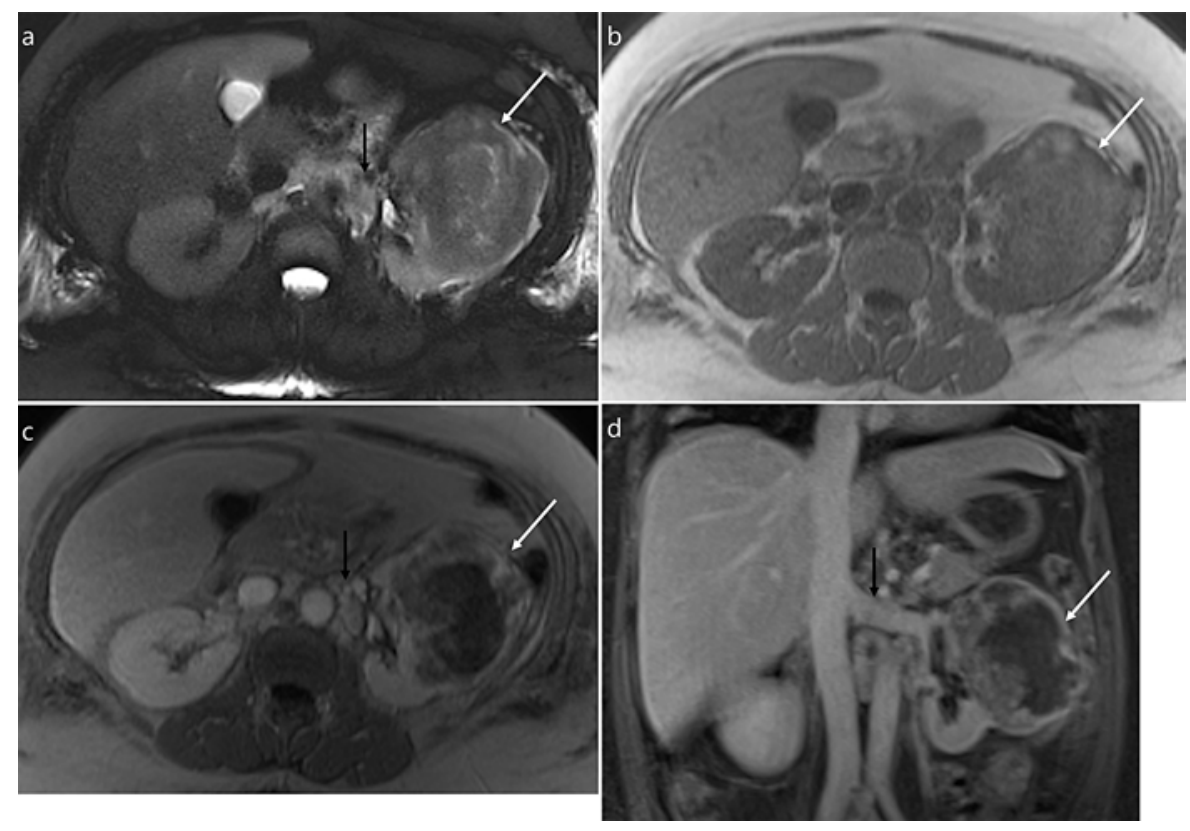

Fig. 1. Transverse T2-weighted single-shot echo train spin echo fat-suppressed MR image (a), transverse T1-weighted magnetization prepared rapid gradient echo in-phase MR image (b), transverse post-gadolinium T1-weighted magnetization prepared rapid gradient echo in-phase MR image (c), and coronal post-gadolinium T1-weighted 3-dimensional gradient echo fat-suppressed MR image (d) show a large renal heterogeneous enhancing mass arising from the left kidney (white arrows, a-d). The mass shows T1-hyperintense proteinaceous/hemorrhagic component and significant central internal necrotic components. Additionally, there are multiple enlarged enhancing retroperitoneal metastatic lymph nodes (black arrows; a, c). The left renal vein is patent (black arrow; d).

surgical intervention, or nephrectomy were determined to be unsafe. Stereotactic radiosurgery (SRS) was considered for the brain metastases but was deferred during the admission due to the small size of the lesions. She was discharged on anticoagulation with an outpatient plan for SRS and consideration for starting systemic therapy for advanced RCC.

The following week, her platelet count was $11 \times 10^{9} / \mathrm{L}$ at the time of outpatient followup. Anticoagulation was held and she was hospitalized for evaluation of the new-onset severe thrombocytopenia. Workup revealed negative heparin-induced thrombocytopenia (HIT)-PF4 antibody test, negative heparin-induced platelet aggregation assay, and normal ADAMTS13 activity. Peripheral blood smear revealed occasional schistocytes; however, disseminated intravascular coagulation (DIC) was felt to be unlikely based on absence of clinical evidence for DIC and a normal fibrinogen level. Given concern for secondary ITP as a paraneoplastic syndrome associated with RCC, dexamethasone $40 \mathrm{mg}$ daily was started for 4 days along with subcutaneous romiplostim ( $1 \mu \mathrm{g}$ per kg weekly), a thrombopoietin mimetic used in treating ITP that stimulates bone marrow production of platelets by megakaryocytes [5].

She achieved a partial response with improvement in platelet count to $76 \times 10^{9} / \mathrm{L}$ after 4 days of dexamethasone and a single romiplostim treatment (Fig. 2). Severe thrombocytopenia recurred within 2 days of completion of dexamethasone. Prednisone $80 \mathrm{mg}$ daily was started and the dose of romiplostim was increased to $3 \mu \mathrm{g} / \mathrm{kg}$ for the next dose (week 2 of treatment); and the platelet count stabilized at $55 \times 10^{9} / \mathrm{L}$. The hospital course was complicated by development of a left upper extremity proximal deep vein thrombosis. Anticoagulation with low-dose enoxaparin $30 \mathrm{mg}$ twice daily was initiated but was periodically held

\section{Karger'}




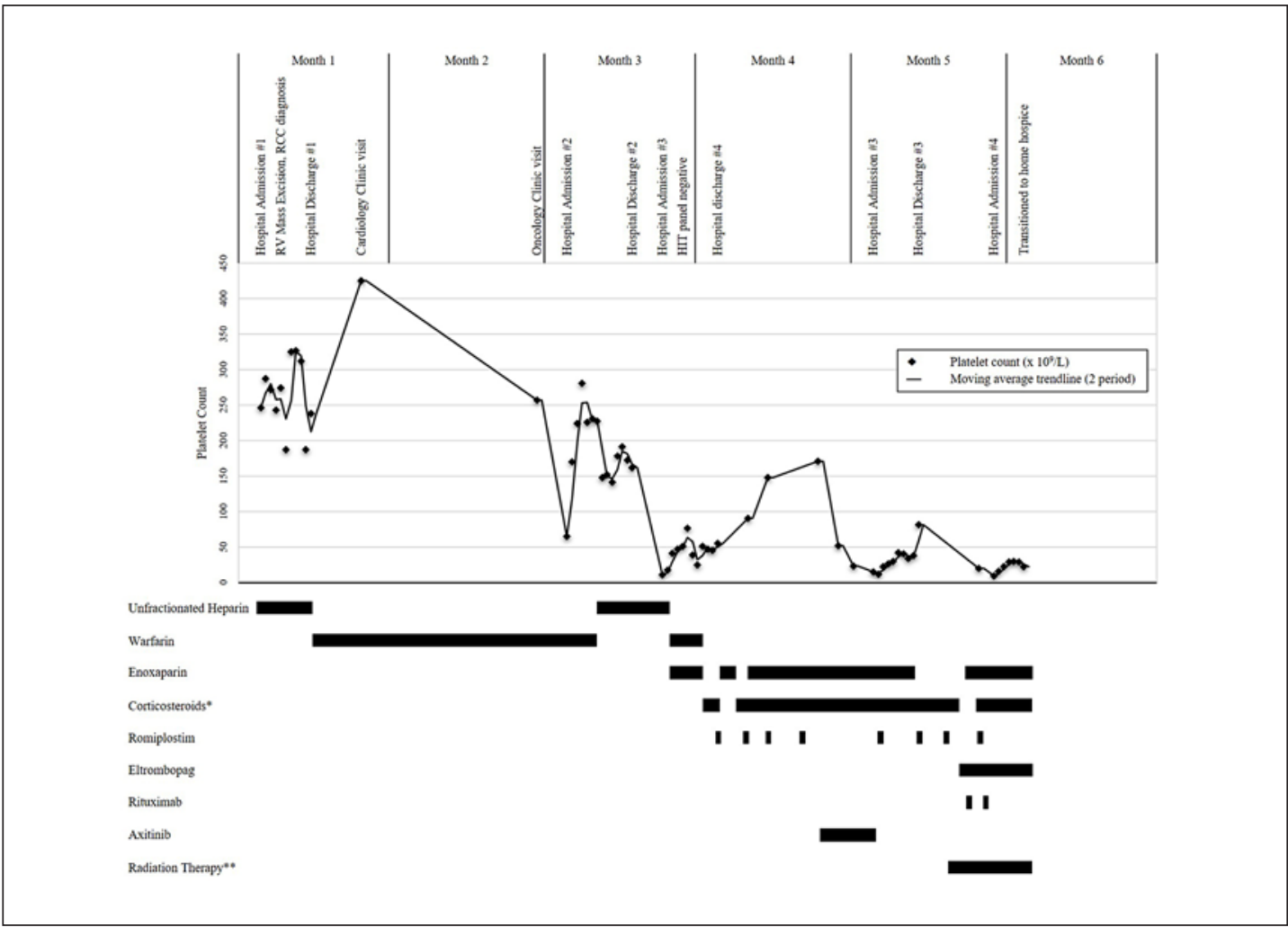

Fig. 2. Platelet trend with associated treatment interventions and notable events. * Corticosteroid treatments included dexamethasone, prednisone, and methylprednisolone. ${ }^{* *}$ Radiation therapy (RT) included whole brain RT (35 Gy), right ventricle RT (30 Gy), and left kidney RT (30 Gy).

during the hospitalization for platelet counts less than $40 \times 10^{9} / \mathrm{L}$. At discharge, enoxaparin was increased to $40 \mathrm{mg}$ twice daily, romiplostim $4 \mu \mathrm{g} / \mathrm{kg}$ was administered, and prednisone was decreased to $60 \mathrm{mg}$ daily. Outpatient plans were made to progressively increase romiplostim dosing and taper prednisone to maintain platelet counts high enough for anticoagulation without the need for steroids if possible, in order to receive systemic therapy for metastatic RCC.

At a 1-week follow-up appointment, the platelet count increased to $148 \times 10^{9} / \mathrm{L}$. Enoxaparin was increased to $60 \mathrm{mg}$ twice daily and prednisone dose taper was initiated. RCCdirected treatment was initiated with axitinib, a small-molecule tyrosine kinase inhibitor, with plans to start pembrolizumab, a programmed death 1 immune checkpoint inhibitor, following SRS for brain metastases and completion of the prednisone taper. Ten days after starting axitinib, follow-up brain MRI prior to SRS demonstrated at least 15 new brain metastases. Whole brain radiation therapy (WBRT) was planned instead of SRS, and axitinib was discontinued. Additionally, her platelet count decreased to $52 \times 10^{9} / \mathrm{L}$ with prednisone having been tapered to $10 \mathrm{mg}$ daily while on weekly romiplostim $(2 \mu \mathrm{g} / \mathrm{kg})$. Prednisone was increased to $20 \mathrm{mg}$ daily, and repeat platelet count the following week was further decreased to $23 \times$ $10^{9} / \mathrm{L}$. Romiplostim dose was increased to $4 \mu \mathrm{g} / \mathrm{kg}$ weekly and enoxaparin was held.

On presentation for radiation therapy the following week, she was found to be hypotensive and hypoxic on room air with reported acute-onset dizziness and back pain since the previous evening. Evaluation in the emergency department included chest CT angiogram which demonstrated increased size of the RV mass $(9.1 \times 4.3 \mathrm{~cm}$; Fig. 3) with increased signif- 


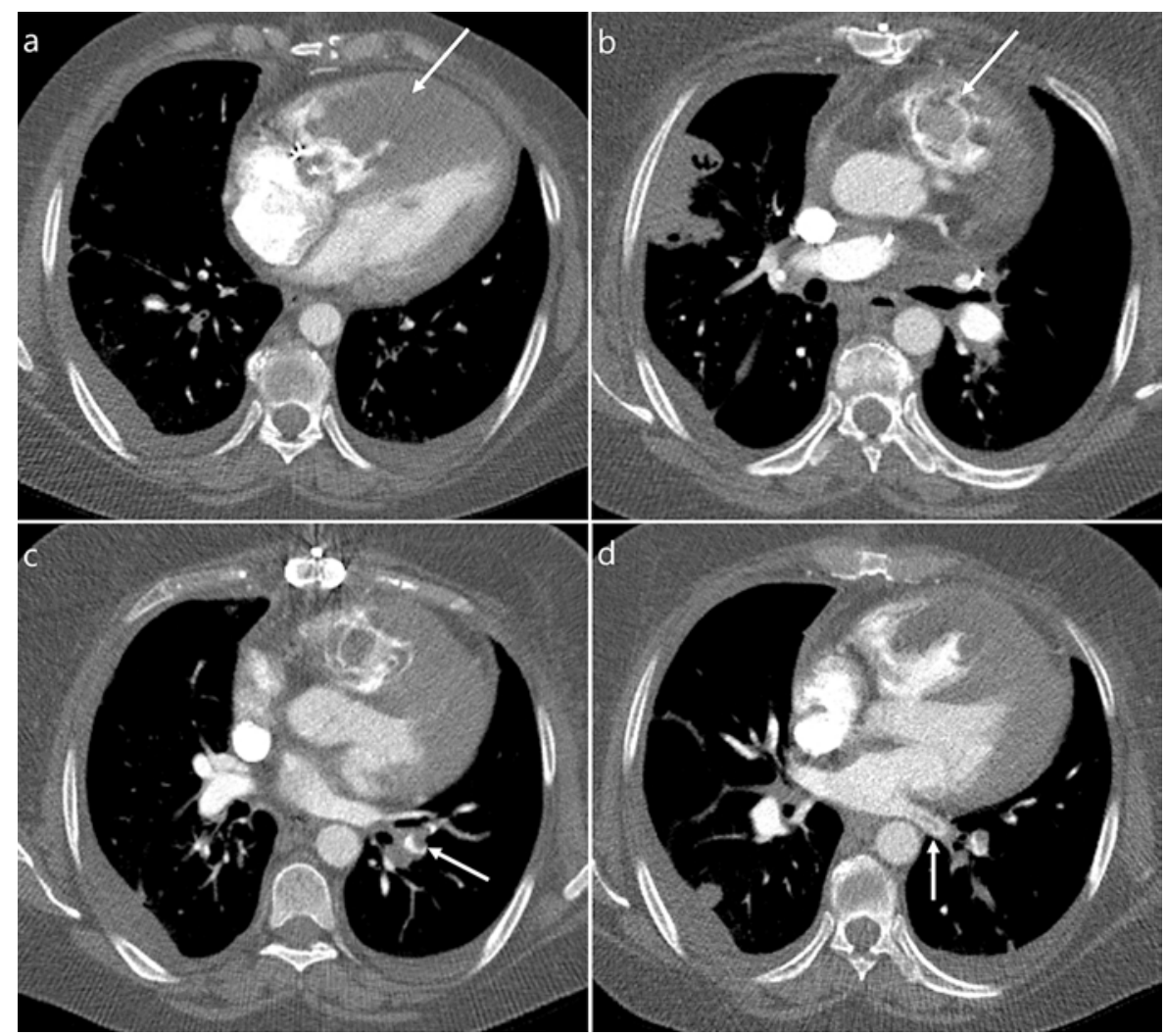

Fig. 3. Transverse images of IV contrast-enhanced chest computed tomography angiography demonstrate a large right ventricular mass (arrow; a) with extension into the right ventricular outflow tract (arrow; b) and associated septal bowing. There are also filling defects in the left lower lobe pulmonary artery (arrow; c) and left inferior pulmonary vein (arrow; d) suggestive of thrombus. Additionally, right lower lobe pulmonary metastatic nodule and bilateral pleural effusions are present (d). Please note the right lung subpleural consolidation and infarct.

icant narrowing of the RV outflow tract, pulmonary emboli similar to the prior scan with a new filling defect of the left lower lobe concerning for additional new pulmonary embolism focus, and a new filling defect in the left inferior pulmonary vein concerning for thrombus. Abdominal/pelvic CT performed concurrently was notable for redemonstration of the large left renal mass with increased retroperitoneal adenopathy. Platelet count was further decreased to $15 \times 10^{9} / \mathrm{L}$. She received intravenous fluids and methylprednisolone $125 \mathrm{mg}$ and was admitted to the medical intensive care unit. Dexamethasone $40 \mathrm{mg}$ daily was resumed and romiplostim $4 \mu \mathrm{g} / \mathrm{kg}$ was continued weekly. Intravenous immunoglobulin (IVIG) was considered at this time but was deferred based on thrombogenic concerns.

Given severe thrombocytopenia precluding systemic therapy and enlargement of the RV tumor thrombus, palliative radiation therapy was planned for the heart and left kidney (30 Gy each) in addition to WBRT (35 Gy). TTE revealed progression of the RV mass to completely fill the RV cavity and cause both RV inflow and outflow obstruction along with flattening of the interventricular septum. Based on continued severe thrombocytopenia and an inability to distinguish her progression of pulmonary emboli as secondary to bland thrombi versus intravascular tumor emboli, anticoagulation was felt to confer more risk than benefit and was held. 
Following minimal response to 4 days of dexamethasone $40 \mathrm{mg}$ daily, second-line treatment with rituximab $375 \mathrm{mg} / \mathrm{m}^{2}$ weekly was added along with increasing romiplostim dosing to $6 \mu \mathrm{g} / \mathrm{kg}$ weekly. Enoxaparin was resumed at $40 \mathrm{mg}$ twice daily once the platelet count stabilized above $30 \times 10^{9} / \mathrm{L}$ and dexamethasone $4 \mathrm{mg}$ twice daily with a taper plan was continued on discharge along with ongoing WBRT.

Despite treatment with 2 doses of rituximab and continued weekly romiplostim, she continued to have severe thrombocytopenia with platelet count nadir at $9 \times 10^{9} / \mathrm{L}$ within a week of completing the dexamethasone taper necessitating rehospitalization. Enoxaparin was once again held and eltrombopag, an oral thrombopoietin receptor agonist (TPO-RA) also used as second-line ITP treatment [6], was added at $75 \mathrm{mg}$ daily in addition to resuming dexamethasone $40 \mathrm{mg}$ daily. During the hospitalization, she developed abdominal pain with elevations in serum hepatic enzymes and hyperbilirubinemia. Magnetic resonance cholangiopancreatography (MRCP) revealed an enhancing soft tissue mass encasing the common bile duct with high-grade stenosis consistent with progressive metastatic disease. Given the refractory thrombocytopenia and metastatic disease progression, she transitioned to hospice care and expired 5 months after initial diagnosis.

\section{Discussion}

For newly diagnosed adult patients with primary ITP, the American Society of Hematology recommends treatment rather than observation for clinically significant bleeding or for platelet counts $<30 \times 10^{9} / \mathrm{L}$ [6]. Standard initial therapy for primary ITP consists of corticosteroids (high-dose dexamethasone or prednisone), IVIG or IV anti-D immunoglobulin (anti-D), with combination treatment and platelet transfusions used for emergent bleeding [6]. While these initial therapies may produce a complete response (CR) as defined by platelet count $\geq 100 \times 10^{9} / \mathrm{L}$ and absence of bleeding [2], additional courses of corticosteroids for relapses are often necessary [6]. IVIG and anti-D are used as adjunctive treatments for patients without significant response to corticosteroids or as primary treatment for patients with a contraindication to corticosteroid use [6].

Given the substantial toxicity of long-term corticosteroid use, second-line treatments including splenectomy, rituximab, and TPO-RAs may be required for patients requiring repeated courses to maintain sufficient platelet counts [6]. Splenectomy has demonstrated the highest cure rate among second-line therapies with a systematic review of 1,223 patients identifying a $72 \%$ success rate at 5 years postoperatively; however, the potential for success must be balanced with the surgical candidacy of the patient [7]. Rituximab, a monoclonal antibody targeting the CD20 protein on B cells, offers an appealing alternative for patients who have failed initial therapy and splenectomy or who are not surgical candidates. Rituximab is administered as a course of 4 weekly infusions, has a favorable side effect profile, and has demonstrated CR in $44 \%$ of patients [8]; however, only 33\% had a platelet count $>50 \times 10^{9} / \mathrm{L}$ at 2 years following a single course [9]. Although TPO-RAs including romiplostim, eltrombopag, and avatrombopag increase platelet counts in patients with persistent severe thrombocytopenia despite prior therapies, chronic maintenance therapy is typically required and studies have not demonstrated reductions in significant bleeding events or total adverse events compared to placebo or standard of care [10]. Fostamatinib, a therapeutic prodrug of a spleen tyrosine kinase (Syk) inhibitor currently under investigation, has shown significant clinical responses in patients resistant to other standard therapies in 2 phase III RCTs [11], although long-term durable response rates remain unknown.

Paraneoplastic ITP has been reported with nearly all types of solid cancers [3]. Although lung and breast cancers have the highest reported cases of ITP, renal and ovarian cancers 
appear to have a higher relative rate of associated ITP as compared to other solid tumor malignancies [3]. In one report of 68 paraneoplastic ITP cases, ITP was seen in most patients either prior to cancer diagnosis (in 17 cases) or concurrently with the identification of the underlying malignancy (in 35 cases) [3]. Based on the outcomes seen in this limited series, the authors concluded that primary tumor resection should be performed in nonmetastatic cases when possible, whereas steroids are likely the best treatment option for patients with metastatic disease [3].

Although less common than other paraneoplastic syndromes associated with RCC, secondary ITP has been described in the literature [3]. First-line therapy for RCC-associated ITP typically is aimed at treatment of the underlying malignancy. Complete responses have been achieved with nephrectomy with and without concurrent splenectomy, although preoperative treatment with steroids and/or IVIG was necessary in some cases [3]. Additionally, one case of ITP refractory to steroids and IVIG demonstrated a response to danazol prior to definitive treatment with nephrectomy [12].

Similar to our patient, management of RCC-associated ITP has been more challenging in cases when nephrectomy was not feasible. In one case report, a patient with RCC had improvement of thrombocytopenia after resection of a metastatic RV mass; however, within 3 weeks of surgery, the platelet count returned to the preoperative levels with recurrence of the mass and concurrent clinical deterioration [13]. An additional case described a patient with limited response to steroids and IVIG after presenting with metastatic RCC and suspected ITP with subsequent intolerance to ipilimumab and nivolumab prior to transitioning to comfort-focused care [14]. One report described achieving CR in a patient with metastatic RCC and ITP refractory to steroids and IVIG after treatment with everolimus [15]. As evidenced by these limited reports, there is a notable lack of clinical data or proven effective treatment approach for RCC-associated ITP, especially in patients that are not candidates for nephrectomy.

\section{Conclusion}

Secondary ITP as a paraneoplastic phenomenon in RCC and other solid tumor malignancies can present significant clinical challenges. In this report, we have described one of the only known cases of RCC-associated ITP refractory to both standard and novel medical therapies including corticosteroids, TPO-RAs, and rituximab along with cancer-directed therapy with axitinib and radiation therapy. While standard therapies for ITP may temporarily improve platelet levels in select patients with RCC, surgical interventions including nephrectomy and/or splenectomy should be considered if possible. Although newer secondline therapies such as rituximab and TPO-RAs may benefit patients with primary ITP, malignancy-associated ITP may prove less responsive, although additional studies are needed to better understand the role of the newer agents in this setting.

\section{Statement of Ethics}

The University of North Carolina Office of Human Research Ethics determined written informed consent from the patient or her family, for the publication of this case, was not required based on the de-identification of all information and the passing of the patient. This submitted case (Reference ID: 309910, IRB Study Number: 20-2397) was reviewed by the Office of Human Research Ethics and was determined to not constitute human subjects research and did not require IRB approval.

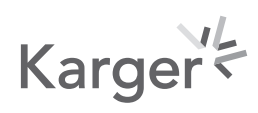




\section{Conflict of Interest Statement}

The authors have no conflicts of interest to disclose.

\section{Funding Sources}

None.

\section{Author Contributions}

All authors provided clinical care for the subject of this case report and contributed substantially to the development of this article. Dr. Dillon C. Cockrell developed the initial draft and it was critically revised based on detailed reviews by Dr. Raj S. Kasthuri, Dr. Ersan Altun, Dr. Tracy L. Rose, and Dr. Matthew I. Milowsky. All authors agreed on the final submission of the manuscript.

\section{References}

1 Terrell DR, Beebe LA, Vesely SK, Neas BR, Segal JB, George JN. The incidence of immune thrombocytopenic purpura in children and adults: A critical review of published reports. Am J Hematol. 2010;85(3):174-80.

2 Rodeghiero F, Stasi R, Gernsheimer T, Michel M, Provan D, Arnold DM, et al. Standardization of terminology, definitions and outcome criteria in immune thrombocytopenic purpura of adults and children: report from an international working group. Blood. 2009 Mar 12;113(11):2386-93.

3 Krauth MT, Puthenparambil J, Lechner K. Paraneoplastic autoimmune thrombocytopenia in solid tumors. Crit Rev Oncol Hematol. 2012 Jan;81(1):75-81.

4 Gold PJ, Fefer A, Thompson JA. Paraneoplastic manifestations of renal cell carcinoma. Semin Urol Oncol. 1996 Nov;14(4):216-22.

5 Hubulashvili D, Marzella N. Romiplostim (Nplate), a treatment option for immune (idiopathic) thrombocytopenic purpura. P T. 2009 Sep;34(9):482-5.

6 Neunert C, Terrell DR, Arnold DM, Buchanan G, Cines DB, Cooper N, et al. American Society of Hematology 2019 guidelines for immune thrombocytopenia. Blood Adv. 2019 Dec 10;3(23):3829-66.

7 Mikhael J, Northridge K, Lindquist K, Kessler C, Deuson R, Danese M. Short-term and long-term failure of laparoscopic splenectomy in adult immune thrombocytopenic purpura patients: a systematic review. Am J Hematol. 2009 Nov;84(11):743-8.

8 Arnold DM, Dentali F, Crowther MA, Meyer RM, Cook RJ, Sigouin C, et al. Systematic review: efficacy and safety of rituximab for adults with idiopathic thrombocytopenic purpura. Ann Intern Med. 2007 Jan 2;146(1):25-33.

9 Godeau B, Porcher R, Fain O, Lefrère F, Fenaux P, Cheze S, et al. Rituximab efficacy and safety in adult splenectomy candidates with chronic immune thrombocytopenic purpura: results of a prospective multicenter phase 2 study. Blood. 2008 Aug 15;112(4):999-1004.

10 Zeng Y, Duan X, Xu J, Ni X. TPO receptor agonist for chronic idiopathic thrombocytopenic purpura. Cochrane Database Syst Rev. 2011 Jul 6(7):CD008235.

11 Bussel J, Arnold DM, Grossbard E, Mayer J, Treliński J, Homenda W, et al. Fostamatinib for the treatment of adult persistent and chronic immune thrombocytopenia: Results of two phase 3, randomized, placebocontrolled trials. Am J Hematol. 2018 Jul; 93(7):921-30.

12 Cho EJ, Lee HJ, Park KU, Kim UY, Lee HJ, Bang SM. Immune thrombocytopenic purpura in a patient with renal cell carcinoma. Korean J Intern Med. 2014 Sep;29(5):671-4.

13 Hasegawa J, Kadoba K, Maruhashi H. Metastatic renal cell carcinoma to the right ventricle complicating thrombocytopenia. J Cardiovasc Surg (Torino). 2002 Apr;43(2):195-7.

14 Noor A, Rehan A, Desai A, Iorgoveanu C, Lee JC. Secondary immune thrombocytopenic purpura in renal cell cancer: a paraneoplastic syndrome. Cureus. 2019 Apr 5;11(4):e4398.

15 Zheng S, Chan H, Epstein RJ, Joseph JE. Resolution of paraneoplastic immune thrombocytopenia following everolimus treatment for metastatic renal cell carcinoma. Intern Med J. 2015 Jun;45(6):666-9.

\section{Karger'k}

